\title{
Prognostic value of the co-expression of carbonic anhydrase IX and vascular endothelial growth factor in patients with clear cell renal cell carcinoma
}

\author{
NGUYEN BA PHUOC ${ }^{1}$, HIDETOSHI EHARA ${ }^{1}$, TAKAHIRO GOTOH ${ }^{1}$, MASAHIRO NAKANO ${ }^{1}$, \\ SHINGO KAMEI ${ }^{1}$, TAKASHI DEGUCHI ${ }^{1}$ and YOSHINOBU HIROSE ${ }^{2}$ \\ ${ }^{1}$ Department of Urology, Gifu University Graduate School of Medicine; \\ ${ }^{2}$ Department of Pathology, Gifu University Hospital, Gifu University, Gifu, Japan
}

Received February 7, 2008; Accepted May 12, 2008

DOI: $10.3892 /$ or_00000037

\begin{abstract}
This study aimed to investigate whether the expression of carbonic anhydrase IX (CAIX) is associated with the expression of vascular endothelial growth factor (VEGF) and whether the co-expression of the two correlates with survival outcome in clear cell renal cell carcinoma (ccRCC). The expression of CAIX and VEGF was evaluated immunohistochemically in 122 paraffin-embedded ccRCC specimens. The clinical significance of these markers in relation to disease-specific survival (DSS) was analyzed. Patients with a low expression of CAIX had a significantly worse prognoses than those with a high expression $(\mathrm{p}=0.0005)$. Inversely, patients with a high expression of VEGF had a significantly worse prognoses than the patients with a low expression $(p=0.0030)$. Furthermore, CAIX expression significantly stratified the DSS of patients with high-stage $(\mathrm{p}=0.0001)$, high-grade $(\mathrm{p}=0.0392)$, low-grade $(\mathrm{p}=0.0273)$, metastasis $(\mathrm{p}=0.0034)$, no metastasis $(\mathrm{p}=0.0303)$ and ECOG$\mathrm{PS}=0(\mathrm{p}=0.0003)$. VEGF expression significantly predicted the survival of patients with low-grade $(\mathrm{p}=0.0003)$, high-stage $(\mathrm{p}=0.0401)$ and ECOG-PS $=0(\mathrm{p}=0.0063)$. A multivariate Cox regression analysis showed that tumor stage $(\mathrm{p}=0.0054)$, metastasis $(p=0.0193)$, ECOG-PS $(p=0.0065)$ and CAIX expression $(\mathrm{p}=0.0001)$ were independent prognostic factors of DSS. Since CAIX and VEGF expression correlated inversely ( $\mathrm{p}=0.0032$ ), the prognostic value of the co-expression of CAIX-VEGF was evaluated. Multivariate analysis revealed that the co-expression was an independent prognostic factor of DSS $(p=0.0002)$. In addition, the co-expression was able to stratify DSS into three risk groups: high-risk, intermediate-risk and low-risk $(\mathrm{p}<0.0001)$. In patients with
\end{abstract}

Correspondence to: Dr H. Ehara, Department of Urology, Gifu University Graduate School of Medicine, 1-1 Yanagido, Gifu 501-1194, Japan

E-mail: h-ehara@gifu-u.ac.jp

Key words: carbonic anhydrase IX, vascular endothelial growth factor, immunohistochemistry, prognosis, renal cell carcinoma
ccRCC, CAIX and VEGF expression correlated inversely. Independent expression of CAIX and a co-expression of CAIX-VEGF were found to be independent predictors of DSS. Furthermore, the co-expression data for CAIX-VEGF provide more accurate prognostic information than the individual data. This information may be useful for survival prediction and risk stratification of patients with ccRCC.

\section{Introduction}

Renal cell carcinoma (RCC) is the most lethal of the common urological cancers, with $\sim 40 \%$ of patients eventually dying of cancer progression (1). Approximately one third of patients present with metastatic disease and up to $50 \%$ of those treated for localized disease experience a recurrence of the disease (2). Despite recent advances in our understanding of RCC biology, the prognosis of patients with symptoms is generally poor and treatment for these patients is limited due to the resistance of RCC to chemotherapy and hormone therapy (3). As such, an accurate prediction of patient outcome after surgery is essential for counseling and for the selection of a follow-up and adjuvant treatment for patients with RCC.

Clear cell renal cell carcinoma (ccRCC) accounts for $\sim 70-80 \%$ of all cases of RCC (4) and is characterized by a high rate of inactivation of the von Hippel-Lindau (VHL) tumor suppressor gene that leads to the accumulation of the hypoxia-inducible factor (HIF), which induces the expression of hypoxia response genes, including carbonic anhydrase IX (CAIX) and vascular endothelial growth factor (VEGF) (5).

CAIX is a transmembrane carbonic anhydrase that plays an essential role in the regulation of proliferation in the response to hypoxic conditions and may be involved in tumor progression (6). CAIX was highly expressed in RCC and was shown as a potential prognostic marker $(7,8)$.

VEGF, a dimeric glycoprotein and a member of the platelet-derived growth factor (PDGF) superfamily of growth factors, plays a critical role in normal and tumor-associated angiogenesis through increased microvascular permeability to plasma proteins, the induction of endothelial cell division and migration and promotion of endothelial cell survival through protection from apoptosis (5). VEGF is one of the most well-studied markers of tumor angiogenesis and its 
expression has been shown to be of prognostic significance in RCC, including ccRCC (9-13). In addition, the overexpression of VEGF may be associated with markers of hypoxia (14).

Therefore, the purpose of our study was to assess whether the expression of CAIX is associated with the expression of VEGF and to test the respective prognostic value of the coexpression of the two in patients with ccRCC.

\section{Materials and methods}

Patients. The study cohort included 122 patients who underwent a radical nephrectomy for ccRCC at Gifu University Hospital between 1987 and 2003. Their outcomes were assessed by a review of clinical and pathological data from hospital records. The tumors were staged according to the 2002 UICC TNM classification of malignant tumors (15) and graded according to the Fuhrman grading scheme (16). Performance status was assessed on the basis of the Eastern Cooperative Oncology Group Performance Score (ECOG-PS) criteria. No patients received treatment for their cancer prior to surgery and salvage interferon- $\alpha$ therapy was used for recurrence.

Sample selection. Archival paraffin-embedded tissue sections of 122 patients were obtained from the Department of Pathology at Gifu University Hospital. Representative hematoxylin and eosin-stained specimens were reviewed in order to confirm the histology and grade and to select sections for immunohistochemical analyses. All tumors were categorized as the clear cell subtype according to the AJCC/UICC classifications. Tissue blocks containing tumor tissue and adjacent normal kidney tissue specimens from each patient were selected and sections were cut to a $4-\mu \mathrm{m}$ thickness and mounted on silane-coated glass slides.

Immunohistochemistry. The rabbit polyclonal antibody against CAIX (H-120, 1:50, Santa Cruz Biotechnology, Santa Cruz, CA, USA) and mouse monoclonal antibody against VEGF (VG1, 1:200, NeoMarkers, Lab Vision Corp., Fremont, CA, USA) were used to detect the CAIX and VEGF protein. Immunohistochemical staining of tissue specimens with antibodies was performed using a peroxidase technique with antigen retrieval using heat treatment, as previously described (9).

The normal kidney tissue adjacent to the tumor in all the specimens was used as an internal control and known positive tumor sections were used as a positive control. As a negative control, we used phosphate-buffered saline (PBS) or normal rabbit immunoglobulin fraction (Dako, Glostrup, Denmark) instead of a primary antibody. The rabbit immunoglobulin fraction was diluted to the same protein concentration as the primary antibodies.

Immunostaining was semi-quantitatively assessed by two investigators (N.B.P. and E.H.) and a pathologist who were unaware of the clinical outcome of each patient. Differences in opinion were resolved based on a common reading. The immunohistochemical results were semi-quantitative, based on the percentage of the positive cells seen in a total field of a single section at $\mathrm{x} 400$ magnification. The pattern of staining was scored as: (0) when $<10 \%$ of the cells stained,
Table I. Characteristics of patients $(n=122)$.

\begin{tabular}{|c|c|}
\hline Variables & Number of patients (\%) \\
\hline \multicolumn{2}{|l|}{ Age (years) } \\
\hline Range & $23-86$ \\
\hline Median & 60 \\
\hline Male/female ratio & $84 / 38$ \\
\hline \multicolumn{2}{|l|}{ ECOG-PS } \\
\hline 0 & $106(86.9)$ \\
\hline 1 & $9(7.4)$ \\
\hline 2 & $6(4.9)$ \\
\hline 3 & $1(0.8)$ \\
\hline \multicolumn{2}{|l|}{ Tumor size (cm) } \\
\hline Range & $1-21.5$ \\
\hline Median & 6.4 \\
\hline$\geq 7$ & $53(43.5)$ \\
\hline$<7$ & $69(56.5)$ \\
\hline \multicolumn{2}{|l|}{ T stage } \\
\hline $\mathrm{T} 1$ & $57(46.7)$ \\
\hline $\mathrm{T} 2$ & $18(14.8)$ \\
\hline $\mathrm{T} 3$ & $17(13.9)$ \\
\hline $\mathrm{T} 4$ & $30(24.6)$ \\
\hline \multicolumn{2}{|l|}{ Grade } \\
\hline G1 & $27(22.1)$ \\
\hline $\mathrm{G} 2$ & $71(58.2)$ \\
\hline G3 & $20(16.4)$ \\
\hline G4 & $4(3.3)$ \\
\hline \multicolumn{2}{|l|}{ Metastasis } \\
\hline Yes & $29(23.7)$ \\
\hline No & $93(76.3)$ \\
\hline Alive & $76(62.3)$ \\
\hline \multicolumn{2}{|l|}{ Died } \\
\hline Cancer & $37(30.3)$ \\
\hline Other causes & $09(7.4)$ \\
\hline \multicolumn{2}{|l|}{ Follow-up (months) } \\
\hline Range & $3.6-240.4$ \\
\hline Median & 69 \\
\hline
\end{tabular}

ECOG-PS, Eastern Cooperative Oncology Group performance status.

(1+) when $10-25 \%$ of the cells stained, $(2+)$ when $25-50 \%$ of the cells stained, $(3+)$ when $50-75 \%$ of the cells stained and (4+) when $>75 \%$ of the cells stained.

Statistical analysis. The follow-up time was defined as the time from surgery to the time of death or the time of the last recorded clinical data. The follow-up examination study endpoint was disease-specific survival (DSS). We used Pearson's Chi-square test to assess the relationship between categorical variables. Survival rates were calculated by the Kaplan-Meier method and the difference between stratified survival functions was analyzed by the log-rank test. The Cox proportional hazards model was used to test the statistical independence and significance of the marker expression in 


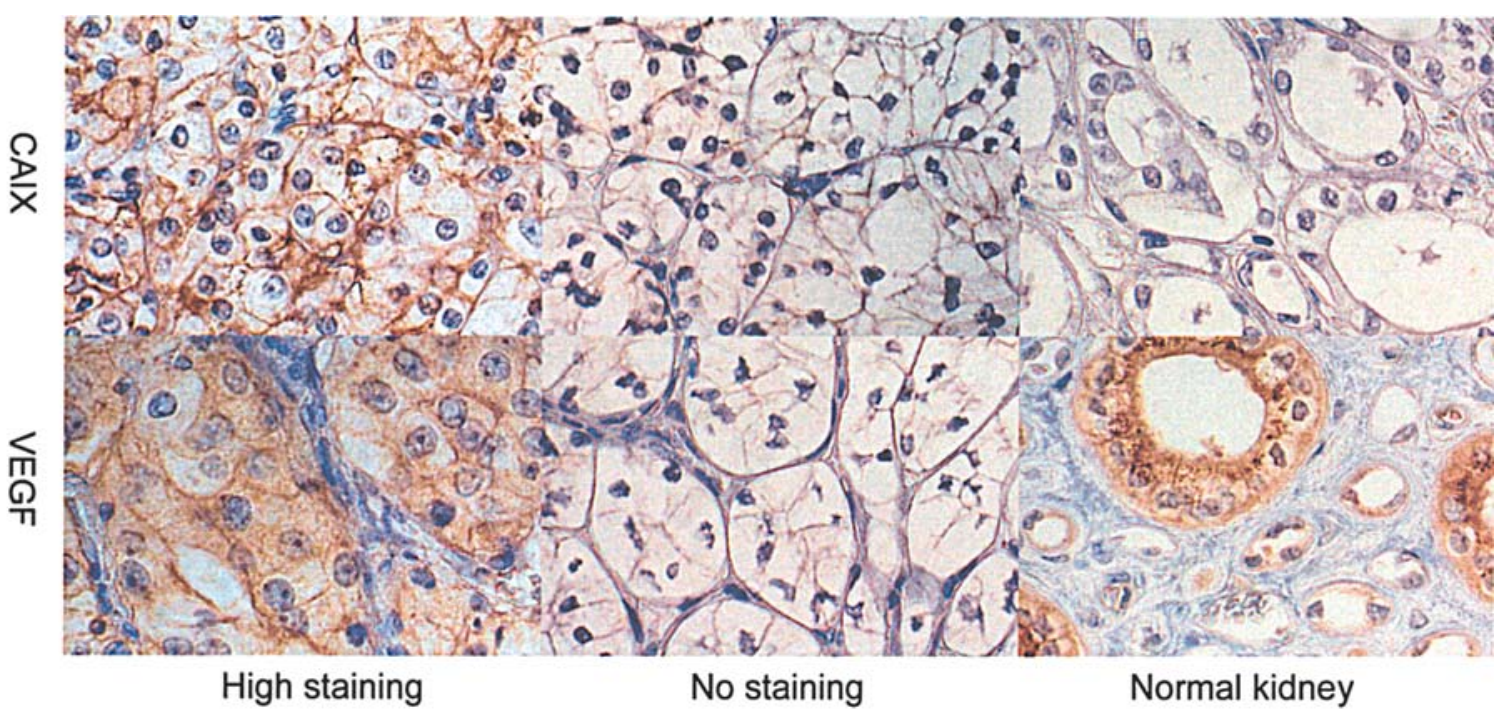

Figure 1. Immunostaining for CAIX and VEGF in ccRCC (both x400 magnification).

Table II. Cox univariate analyses of the disease-specific survival in all cases $(n=122)$.

\begin{tabular}{lcccc}
\hline Variable & Categories & Risk ratio & $95 \% \mathrm{CI}$ & P-value \\
\hline Age $(\mathrm{yr})$ & $\geq 60$ vs. $<60$ & 1.807 & $0.913-3.579$ & 0.0980 \\
Sex & Male vs. Female & 0.816 & $0.395-1.688$ & 0.5842 \\
Tumor size (cm) & $\geq 7$ vs. $<7$ & 4.362 & $2.104-9.042$ & $0.0001^{\mathrm{a}}$ \\
ECOG-PS & 0 vs. $>0$ & 3.540 & $1.692-7.406$ & $0.0025^{\mathrm{a}}$ \\
T stage & T1/T2 vs. T3/T4 & 9.814 & $4.294-22.42$ & $<0.0001^{\mathrm{a}}$ \\
Grade & G1/G2 vs. G3/G4 & 4.497 & $2.245-9.006$ & $<0.0001^{\mathrm{a}}$ \\
Metastasis & Yes vs. No & 0.097 & $0.049-0.190$ & $<0.0001^{\mathrm{a}}$ \\
CAIX & High vs. Low & 0.330 & $0.172-0.634$ & $0.0013^{\mathrm{a}}$ \\
VEGF & High vs. Low & 2.618 & $1.357-5.053$ & $0.0046^{\mathrm{a}}$ \\
CAIX-VEGF & High-Inte-Low & 2.285 & $1.514-3.449$ & $0.0001^{\mathrm{a}}$ \\
\hline
\end{tabular}

CI, confidence interval; High, high-risk; Inte, intermediate-risk; Low, low-risk; ECOG-PS, Eastern Cooperative Oncology Group performance

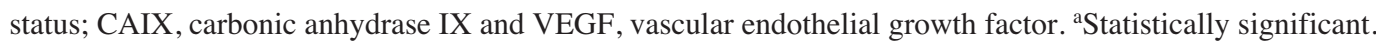

predicting survival or the risk of patient death based on a variety of potential prognostic factors. The Schoenfeld residual test was used to evaluate the proportional hazard assumptions. A $p<0.05$ was considered statistically significant. Variables that were shown by the univariate analysis to influence survival significantly were entered into the multivariate Cox regression model. All statistical analyses were performed using a commercially available software program (Statview, Version 5.0, Abacus Concepts, Berkeley, CA, USA).

\section{Results}

Characteristics of patients. The clinicopathological characteristics and outcomes of 122 patients are shown in Table I. During the follow-up period, 37 (30.3\%) patients died of ccRCC (median survival, 37.8 months) and nine (7.4\%) died of other causes (median survival, 70.8 months). Twenty- nine patients had metastasis at the time of surgery and only one patient without metastasis at the time of surgery had lymph node invasion. Therefore, the nodal status was considered to reflect the metastatic status in this study.

Immunostaining. The immunostaining of CAIX was not observed in adjacent normal kidney tissue. VEGF was expressed in the cytoplasm of tubular cells, though not glomerular or vascular endothelial cells. Immunostaining of CAIX was observed predominantly in the cell membrane and that of VEGF was detected in the cytoplasm and cell membrane of the cancer cells (Fig. 1).

Clinical correlation and survival prognosis. For a survival analysis, the expression of markers was categorized into two groups, either high expression (CAIX expression score $4^{+}$or VEGF expression score $3^{+}$and $4^{+}$) or low expression (CAIX 
expression $<4^{+}$score, VEGF expression $<3^{+}$score $)$. The cutoff level of the marker was selected according to the receiver operating characteristic curve.

A high expression of CAIX and VEGF was detected in $87(71.3 \%)$ and $45(36.8 \%)$ of the 122 ccRCCs, respectively. The expression of CAIX was not associated with any clinicopathological parameter, whereas the expression of VEGF was associated with the tumor stage $(p=0.0081)$ and metastasis $(\mathrm{p}=0.0079)$. The expression of CAIX correlated inversely with the expression of VEGF $(p=0.0032)$. The Kaplan-Meier-estimated DSS (Fig. 2) revealed that patients $(n=35)$ with a low expression of CAIX had significantly worse prognoses than those $(n=87)$ with a high expression $(\mathrm{p}=0.0005)$. Inversely, patients $(\mathrm{n}=45)$ with a high expression of VEGF had significantly worse prognoses than patients $(\mathrm{n}=77)$ with a low expression $(\mathrm{p}=0.0030)$. More specifically, the expression of CAIX significantly stratified the survival of patients with high tumor stage $(\mathrm{p}=0.0001)$, tumor grade $1-2$ $(\mathrm{p}=0.0273)$, tumor grade $3-4(\mathrm{p}=0.0392)$, metastasis $(\mathrm{p}=0.0034)$, no metastasis $(\mathrm{p}=0.0303)$ and ECOG-PS $=0$ $(\mathrm{p}=0.0003)$. The expression of VEGF significantly predicted the survival in patients with tumor grade $1-2(\mathrm{p}=0.0003)$, high tumor stage $(p=0.0401)$, and ECOG-PS $=0 \quad(p=0.0063)$. A univariate analysis of the different clinicopathological factors and tumor markers confirmed that the expression of CAIX and VEGF, tumor stage, tumor grade, metastatic status, tumor size and ECOG-PS were all statistically significant prognostic factors (Table II). The multivariate Cox regression analysis showed the tumor stage $(\mathrm{p}=0.0054)$, metastatic status $(\mathrm{p}=0.0193)$, ECOG-PS $(\mathrm{p}=0.0065)$ and expression of CAIX ( $\mathrm{p}=0.0001)$ to be independent prognostic factors of DSS (Table III).

Since the expression of CAIX correlated inversely with the expression of VEGF ( $p=0.0032$ ), the expression of these two biomarkers was incorporated into a single prognostic marker, which was able to stratify DSS into three risk groups. The high-risk group included tumors with a low expression of CAIX and a high expression of VEGF. The intermediate risk group included tumor specimens that had a high expression of CAIX and VEGF or a low expression of both CAIX and VEGF (the survival of patients with a high expression of CAIX and VEGF did not differ statistically from that of patients with a low expression of CAIX and VEGF, $\mathrm{p}=0.7925$, median DSS: 58.3 vs. 60.6 months). The low-risk group included tumor specimens with a high expression of CAIX and a low expression of VEGF. Kaplan-Meier curves for each risk group were significantly different from each other ( $<<0.0001$, Fig. 2C). A stepwise multivariate analysis revealed that the co-expression of CAIX-VEGF was an independent prognostic factor of DSS ( $p=0.0002$, Table III).

\section{Discussion}

The recent identification and potential incorporation of molecular markers in the diagnosis and prognostication of $\mathrm{RCC}$ are expected to play an important role in the selection of patients for adjuvant therapies and may be used to predict the clinical response to target therapies. The accepted method of clinical validation of novel markers is through formalin-fixed and paraffin-embedded specimens using immunohisto-
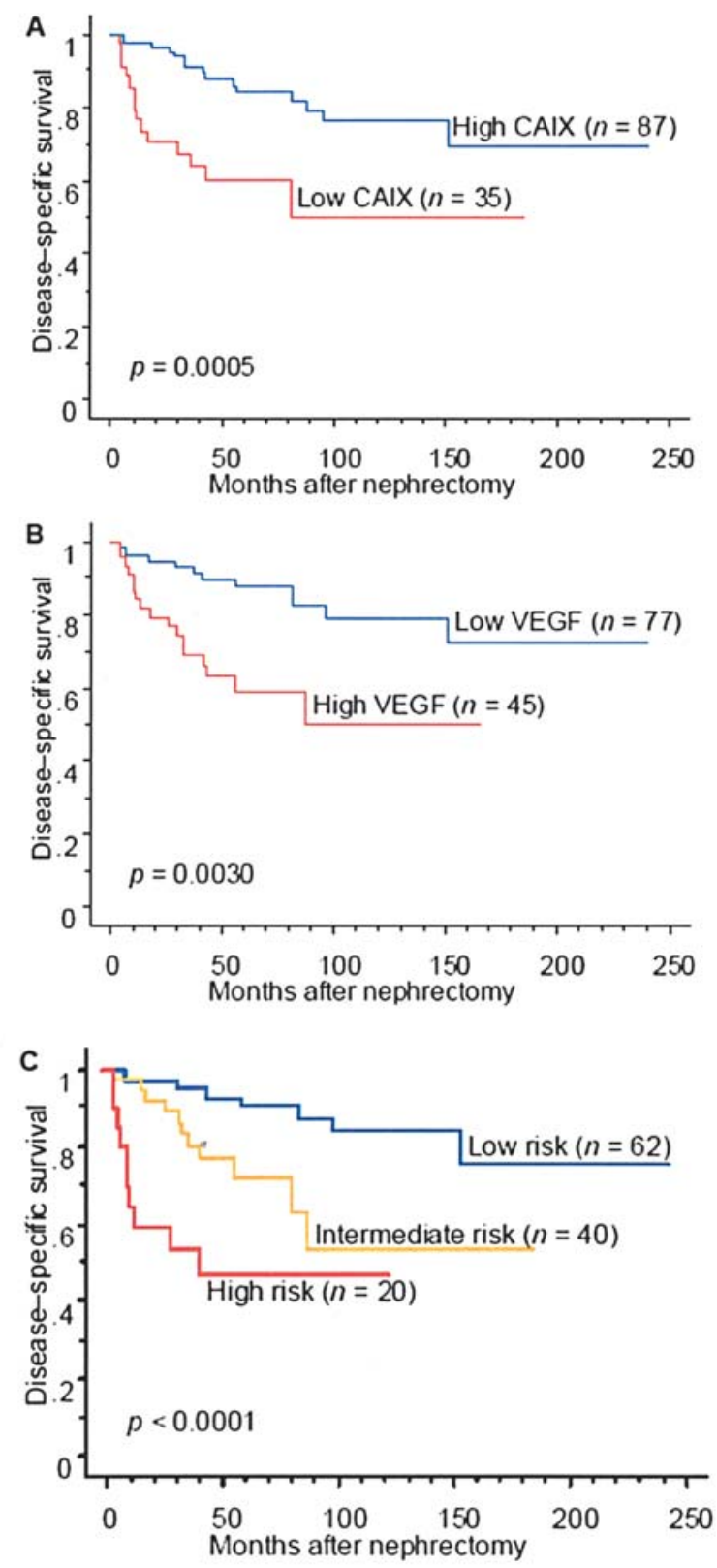

Figure 2. Disease-specific survival in patients with ccRCC. Kaplan-Meier survival curves according to the expression of CAIX (A), VEGF (B) and the co-expression of CAIX-VEGF (C). Blue line, low-level staining for tumor marker or low-risk for the co-expression; red line, high-level staining for tumor markers or high-risk for co-expression; yellow line, intermediate risk for co-expression.

chemistry. This technology has enabled the analysis of protein expression profiles on specimens in order to determine their potential clinical significance and role in RCC biology (17).

In the current study of ccRCC, CAIX and VEGF proteins were detected using immunohistochemistry. Differing from most previous studies, which were conducted using mAb against CAIX, we used polyclonal antibody, CAIX (H-120), to detect the CAIX protein. CAIX (H-120) is specific for the detection of CAIX of human origin by Western blotting and immunohistochemistry with paraffin-embedded sections (data unpublished).

Previous immunohistochemical studies of human tumor tissues using mAb CAIX revealed that CAIX was highly 
Table III. Cox multivariate analyses of the disease-specific survival in all cases $(n=122)$.

\begin{tabular}{|c|c|c|c|c|}
\hline Variable & Categories & Risk ratio & $95 \% \mathrm{CI}$ & P-value \\
\hline Tumor size $(\mathrm{cm})$ & $\geq 7$ vs. $<7$ & 1.530 & $0.674-3.472$ & 0.3090 \\
\hline ECOG-PS & 0 vs. $>0$ & 3.295 & $1.418-7.654$ & $0.0065^{\mathrm{a}}$ \\
\hline T stage & T1/T2 vs. T3/T4 & 5.296 & $1.636-17.14$ & $0.0054^{\mathrm{a}}$ \\
\hline Grade & G1/G2 vs. G3/G4 & 1.104 & $0.498-2.449$ & 0.8069 \\
\hline Metastasis & Yes vs. No & 0.328 & $0.129-0.834$ & $0.0193^{\mathrm{a}}$ \\
\hline CAIX & High vs. Low & 0.205 & $0.093-0.452$ & $0.0001^{\mathrm{a}}$ \\
\hline VEGF & High vs. Low & 1.173 & $0.553-2.489$ & 0.6768 \\
\hline \multicolumn{5}{|c|}{ Combining CAIX and VEGF } \\
\hline Tumor size $(\mathrm{cm})$ & $\geq 7$ vs. $<7$ & 1.675 & $0.733-3.827$ & 0.2213 \\
\hline ECOG-PS & $0 \mathrm{vs} . .>0$ & 2.407 & $1.093-5.302$ & $0.0290^{\mathrm{a}}$ \\
\hline T stage & T1/T2 vs. T3/T4 & 3.924 & $1.237-12.45$ & $0.0196^{\mathrm{a}}$ \\
\hline Grade & G1/G2 vs. G3/G4 & 1.055 & $0.481-2.312$ & 0.8938 \\
\hline Metastasis & Yes vs. No & 0.318 & $0.128-0.795$ & $0.0137^{\mathrm{a}}$ \\
\hline CAIX-VEGF & High-Inte-Low & 2.353 & $1.473-3.3759$ & $0.0002^{\mathrm{a}}$ \\
\hline
\end{tabular}

CI, confidence interval; High, high risk; Inte, intermediate risk; Low, low risk; ECOG-PS, Eastern Cooperative Oncology Group performance status; CAIX, carbonic anhydrase IX and VEGF, vascular endothelial growth factor. a Statistically significant.

expressed in a tumor of the kidney, cervix, breast, lung, esophagus, stomach, and biliary tract, although it is absent in most normal tissue, with the exception of epithelial cells of the gastrointestinal tract and gallbladder (18). In the present study, we found that CAIX is expressed at a high level in ccRCC, though is not expressed in normal kidney tissue adjacent to the tumor. Furthermore, the high expression of CAIX is associated with increased survival in ccRCC; these results are consistent with previous findings $(7,8,19,20)$. Studies of cervical and colorectal cancer also found that increased CAIX expression correlated with a good prognosis $(21,22)$. However, studies of other cancers, including breast, esophageal, lung, nasopharyngeal, and astrocytic cancers have found that a high expression of CAIX is associated with a poor prognosis (23-27). It is unclear as to why CAIX overexpression is associated with an adverse prognosis in cancer. It may be that CAIX expression reflects tumor progression or that it directly influences tumor behavior (7). In RCC, CAIX expression may serve as a surrogate for some other critical HIF-mediated protein that is more directly associated with immune responsiveness (20). CAIX expression varies among different kidney tumors. Liao et al (28) demonstrated that CAIX is expressed at a high level in ccRCC and is not expressed in non-ccRCC, thus suggesting that the expression of the CAIX protein may be used as a marker for the differential diagnosis of the ccRCC subtype. Moreover, Atkins et al (20) reported that CAIX expression may serve as a marker in predicting the response of RCC to interleukin-2 therapy. Taken together, the above data suggest that CAIX expression serves as a strong biomarker for RCC and is particularly important for ccRCC.
$\mathrm{RCC}$ has long been recognized as one of the most hypervascularized cancers, as reflected by the distinctive neovascularity exhibited on renal angiography (4). Tumor development requires the induction of angiogenesis. The primary inducer of angiogenesis in ccRCC is VEGF, which is suppressed by the wild-type VHL protein under normal conditions and is dramatically up-regulated during tumor development (5). A high expression of VEGF has been associated with worse survival and an increased incidence of disease recurrence in many malignancies, including RCC (14). In the present study, we showed that VEGF expression significantly correlates with tumor stage and metastasis ( $\mathrm{p}=0.0126$ and 0.0079 , respectively). In addition, our study confirmed the previously reported finding that a high expression of VEGF was a significant predictor of a worse outcome in patients with ccRCC $(9,13,15,16)$. Rini and Small (5) provided compelling evidence for VHL gene inactivation in the majority of ccRCC tumors leading to VEGF overexpression, which promotes tumor angiogenesis, development and growth. Therefore, VEGF may be used as a prognostic factor for ccRCC and ccRCC may be a good target for antiangiogenic approaches to therapy.

The present study evaluated the expression of CAIX and VEGF simultaneously in the same tissue samples from patients with ccRCC. We found that the expression of CAIX and VEGF correlated inversely. Jubb et al examined a large and diverse cohort of tumors and found that CAIX expression correlated with VEGF expression only in the renal tumor (14). Studies on esophageal, nasopharyngeal and lung cancer show that no correlation exists between CAIX and VEGF expressions $(24,26,29)$. The strong association of CAIX 
expression with VEGF expression may result from VHL gene inactivation in the majority of ccRCC. In our study, the expression of CAIX and VEGF was assessed as a single prognostic factor. We found the co-expression of CAIX-VEGF to be an independent prognostic factor of DSS. Furthermore, the co-expression was able to stratify DSS into three risk groups, namely, high-, intermediate- and low-risk. As expected, the predictive accuracy of the co-expression, as estimated by the median DSS, increased in comparison to other prognostic factors.

Based on the genetics and biology of ccRCC, we examined the expression of CAIX and VEGF simultaneously in the same tissue samples from patients with ccRCC by immunohistochemistry. Our data suggest that the correlation of the expression of CAIX and VEGF may be specific to ccRCC and that the co-expression data for CAIX-VEGF provide more accurate prognostic information than the individual expression data in ccRCC. This information may be useful for predicting survival and for identifying the patients who are at high-risk and who would potentially benefit from an adjuvant clinical trial.

\section{References}

1. Jemal A, Siegel R, Ward E, Murray T, Xu J and Thun MJ: Cancer statistics. CA Cancer J Clin 57: 43-66, 2007.

2. Lam JS, Leppert JT, Figlin RA, et al: Surveillance following radical or partial nephrectomy for renal cell carcinoma. Curr Urol Rep 6: 7-18, 2005.

3. Motzer RJ, Mazumdar M, Bacik J, Russo P, Berg WJ and Metz EM: Effect of cytokine therapy on survival for patients with advanced renal cell carcinoma. J Clin Oncol 18: 19281935,2000

4. Novick AC and Campbell SC: Renal tumors. In: Campbell's Urology. WB Saunders (ed). 8th edition, Philadelphia, pp2672-2719, 2002.

5. Rini BI and Small EJ: Biology and clinical development of vascular endothelial growth factor-targeted therapy in renal cell carcinoma. J Clin Oncol 23: 1028-1043, 2005.

6. Ivanov S, Liao SY, Ivanova A, et al: Expression of hypoxiainducible cell-surface transmembrane carbonic anhydrases in human cancer. Am J Pathol 158: 905-919, 2001.

7. Bui MH, Seligson D, Han KR, et al: Carbonic anhydrase IX is an independent predictor of survival in advanced renal clear cell carcinoma: implications for prognosis and therapy. Clin Cancer Res 9: 802-811, 2003.

8. Bui MH, Visapaa H, Seligson D, et al: Prognostic value of Carbonic anhydrase IX and Ki67 as predictors of survival for renal clear cell carcinoma. J Urol 171: 2461-2466, 2004.

9. Phuoc NB, Ehara H, Gotoh T, et al: Immunohistochemical analysis with multiple antibodies in search of prognostic markers for clear cell renal cell carcinoma. Urology 69: 843-848, 2007.

10. Paradis V, Lagha NB, Zeimoura L, et al: Expression of vascular endothelial growth factor in renal cell carcinomas. Virchows Arch 436: 351-356, 2000.

11. Yildiz E, Gokce G, Kilicarslan H, Ayan S, Goze OF and Guitekin EY: Prognostic value of the expression if Ki-67, CD44 and vascular endothelial growth factor, and microvessel invasion, in renal cell carcinoma. BJU Int 93: 1087-1093, 2004.

12. Jacobsen J, Grankvist K, Rasmuson T, Bergh A, Landberg G and Ljungberg B: Expression of vascular endothelial growth factor protein in human renal cell carcinoma. BJU Int 93: 297-302, 2004.
13. Rioux-Leclercq N, Fergelot P, Zerrouki S, et al: Plasma level and tissue expression of vascular endothelial growth factor in renal cell carcinoma: a prospective study of 50 cases. Hum Pathol 38: 1489-1495, 2007.

14. Jubb AM, Pham TQ, Hanby AM, et al: Expression of vascular endothelial growth factor, hypoxia inducible factor 1alpha, and carbonic anhydrase IX in human tumours. J Clin Pathol 57: 504-512, 2004.

15. Sobin LH and Wittekind $\mathrm{CH}$ (eds): International Union Against Cancer. UICC TNM Classification of Malignant Tumours. 6th edition, New York, pp193-195, 2002.

16. Fuhrman SA, Lasky LC and Limas C: Prognostic significance of morphologic parameters in renal cell carcinoma. Am J Surg Pathol 6: 655-663, 1982.

17. Lam JS, Pantuck AJ, Belldegrun AS and Figlin RA: Protein expression profiles in renal cell carcinoma: staging, prognosis, and patient selection for clinical trials. Clin Cancer Res 13: 703-708, 2007.

18. Leppert JT, Lam JS, Pantuck AJ, Figlin RA and Belldegrun AS Carbonic anhydrase IX and the future of molecular markers in renal cell carcinoma. BJU Int 96: 281-285, 2005.

19. Sandlund J, Oosterwijk E, Grankvist K, Oosterwijk-Wakka J, Ljungberg B and Rasmuson T: Prognostic impact of carbonic anhydrase IX expression in human renal cell carcinoma. BJU Int 100: 556-560, 2007

20. Atkins M, Regan M, McDermott D, Mier J, Stanbridge E and Youmans A: Carbonic Anhydrase IX expression predict outcome of interleukin 2 therapy for renal cancer. Clin Cancer Res 11: 3714-3721, 2005.

21. Brewer CA, Liao SY, Wilczynski SP, et al: A study of biomarkers in cervical carcinoma and clinical correlation of the novel biomarker MN. Gynecol Oncol 63: 337-344, 1996.

22. Saarnio J, Parkkila S, Parkkila AK, et al: Immunohistochemical study of colorectal tumors for expression of a novel transmembrane carbonic anhydrase. MN/CA IX, with potential value as a marker of cell proliferation. Am J Pathol 153: 279-285, 1998.

23. Hussain SA, Ganesan R, Reynolds G, et al: Hypoxia-regulated carbonic anhydrase IX expression is associated with poor survival in patients with invasive breast cancer. Br J Cancer 96: 104-109, 2007.

24. Driessen A, Landuyt W, Pastorekova S, et al: Expression of carbonic anhydrase IX (CAIX), a hypoxia-related protein, rather than vascular-endothelial growth factor (VEGF), a proangiogenic factor, correlates with an extremely poor prognosis in esophageal and gastric adenocarcinomas. Ann Surg 243: 334-340, 2006.

25. Giatromanolaki A, Koukourakis MI, Sivridis E, et al: Expression of hypoxia-inducible carbonic anhydrase-9 relates to angiogenic pathways and independently to poor outcome in non-small cell lung cancer. Cancer Res 61: 7992-7998, 2001.

26. Hui EP, Chan AT, Pezzella F, et al: Coexpression of hypoxiainducible factors 1alpha and 2alpha, carbonic anhydrase IX, and vascular endothelial growth factor in nasopharyngeal carcinoma and relationship to survival. Clin Cancer Res 8: 2595-2604, 2002.

27. Haapasalo JA, Nordfors KM, Hilvo M, et al: Expression of carbonic anhydrase IX in astrocytic tumors predicts poor prognosis. Clin Cancer Res 12: 473-477, 2006.

28. Liao SY, Aurelio ON, Jan K, Zavada J and Stanbridge EJ: Identification of the MN/CA9 protein as a reliable diagnostic biomarker of clear cell carcinoma of the kidney. Cancer Res 57: 2827-2831, 1997.

29. Kim SJ, Rabbani ZN, Dewhirst MW, et al: Expression of HIF-1 $\alpha$, CA IX, VEGF, and MMP-9 in surgically resected non-small cell lung cancer. Lung Cancer 49: 325-335, 2005. 